\title{
WOWA operators in fuzzy context sequences
}

\author{
C.Alcalde ${ }^{1}$ A. Burusco B $^{2,3}$ \\ ${ }^{1}$ Dept. of Applied Mathematics. University of the Basque Country UPV/EHU. \\ Plaza de Europa 1, 20018 San Sebastián. Spain. \\ Email: c.alcalde@ehu.es \\ 2 Dept. of Automatics and Computing. Public University of Navarra. \\ Campus de Arrosadía, 31006 Pamplona. Spain. \\ Email: burusco@unavarra.es \\ ${ }^{3}$ Smart Cities Institute, Public University of Navarra
}

\begin{abstract}
In some cases, the relationship between an object set $X$ and an attribute set $Y$ is set up by means of a fuzzy context sequence. A particular case of this situation appears when we want to study the evolution in time of a fuzzy context.

In this work, we deepen in the study of these situations. First we recover the fuzzy context sequence definition and the main results. After that, with the aid of WOWA operators and the $L$-fuzzy concept analysis, we propose a study of the sequences establishing tendencies and we compare the results with the previous obtained. Therefore, the $L$-fuzzy concept analysis can be interpreted as a tool for trend estimation.

Finally, we illustrate all the results by means of an example.
\end{abstract}

Keywords: $L$-fuzzy concept analysis, fuzzy context sequences, WOWA operators.

\section{Introduction}

The $L$-fuzzy Concept Analysis obtain the information from a $L$-fuzzy context by means of the $L$ fuzzy concepts. These $L$-fuzzy contexts are tuples $(L, X, Y, R)$, with $L$ a complete lattice, $X$ and $Y$ sets of objects and attributes, and $R \in L^{X \times Y}$ a $L$-fuzzy relation between the objects and the attributes.

In some situations, we have several relations between the object set $X$ and the attribute set $Y$, making up what we are going to say a fuzzy context sequence. When this sequence represents an evolution in time, we can be more ambitious and try to predict future tendencies besides studying past behaviors. The study of this fuzzy context sequences will be the main target of this work.

We take as starting point a sequence formed by the $L$-fuzzy contexts $\left(L, X, Y, R_{i}\right), i=\{1, \ldots, n\}$, with $n \in \mathbb{N}$, where $X$ and $Y$ are the sets of objects and attributes respectively, $L=[0,1]$ and $R_{i} \in L^{X \times Y}$ represents the $i$ th relation between the objects of $X$ and the attributes of $Y$.

The final goal is the study of the fuzzy context sequences when they represent the evolution in time of the fuzzy contexts.

In this case, it will be of special interest the study of the evolution of the attributes by means of the search of patterns. Works in this line to analyze the course of time in a Formal context can be found in $[18,24,25]$.

In $[24,25]$ K.E. Wolff defines the Temporal Concept Analysis where a Conceptual Time System is introduced such that the state and phase spaces are defined as concept lattices which represent the meaning of the states with respect to the chosen time description. On the other hand, the authors define the hidden evolution patterns in $[18,21]$ using temporal matching in the case of Formal Concept Analysis.

In [1], we showed a study of these fuzzy context sequences using OWA operators. In this work, we are going to improve those results using WOWA operators [22]. In this case, $L$-fuzzy concept analysis can be interpreted as a tool for trend estimation.

The rest of the paper is organized as follows: Section 2 recover the main results of the $L$-fuzzy Concept Analysis. In Section 3 the fuzzy context sequences are analyzed. Then, in Section 4, the evolution in time of these contexts is tackled using WOWA operators. Finally, the conclusions and future work are detailed in Section 5. Furthermore, an interesting example can be found throughout the paper.

\section{2. $L$-fuzzy concept analysis}

The Formal Concept Analysis of R. Wille [23] extracts information from a binary table that represents a Formal context $(X, Y, R)$ with $X$ and $Y$ finite sets of objects and attributes respectively and $R \subseteq X \times Y$. The hidden information consists of pairs $(A, B)$ with $A \subseteq X$ and $B \subseteq Y$, called Formal concepts, verifying $A^{*}=B$ and $B^{*}=A$, where $(\cdot)^{*}$ is the derivation operator that associates the attributes related to the elements of $A$ with every object set $A$, and the objects related to the attributes of $B$ with every attribute set $B$. These Formal Concepts can be interpreted as a group of objects $A$ that shares the attributes of $B$.

In previous works $[6,7]$ we have defined the $L$ - 
fuzzy contexts $(L, X, Y, R)$, with $L$ a complete lattice, $X$ and $Y$ sets of objects and attributes respectively and $R \in L^{X \times Y}$ a fuzzy relation between the objects and the attributes. This is an extension of Wille's Formal contexts to the fuzzy case when we want to study the relations between the objects and the attributes with values in a complete lattice $L$, instead of binary values.

In our case, to work with these $L$-fuzzy contexts, we have defined the derivation operators 1 and 2 given by means of these expressions:

$$
\forall A \in L^{X}, \forall B \in L^{Y}
$$

$$
\begin{aligned}
& A_{1}(y)=\inf _{x \in X}\{\mathcal{I}(A(x), R(x, y))\} \\
& B_{2}(x)=\inf _{y \in Y}\{\mathcal{I}(B(y), R(x, y))\}
\end{aligned}
$$

with $\mathcal{I}$ a fuzzy implication operator defined in the lattice $(L, \leq)$.

The information stored in the context is visualized by means of the $L$-fuzzy concepts that are pairs $\left(M, M_{1}\right) \in L^{X} \times L^{Y}$ with $M \in f i x(\varphi)$, set of fixed points of the operator $\varphi$, being defined from the derivation operators 1 and 2 as $\varphi(M)=\left(M_{1}\right)_{2}=$ $M_{12}$. These pairs, whose first and second components are said to be the fuzzy extension and intension respectively, represent a group of objects that share a group of attributes in a fuzzy way.

Using the usual order relation between fuzzy sets, that is,

$$
\forall M, N \in L^{X}, M \leq N \Longleftrightarrow M(x) \leq N(x), \forall x \in X
$$

we define the set $\mathcal{L}=\left\{\left(M, M_{1}\right) / M \in f i x(\varphi)\right\}$ with the order relation $\preceq$ defined as:

$$
\begin{aligned}
& \forall\left(M, M_{1}\right),\left(N, N_{1}\right) \in \mathcal{L}, \\
& \quad\left(M, M_{1}\right) \preceq\left(N, N_{1}\right) \text { if } M \leq N\left(\text { or } N_{1} \leq M_{1}\right)
\end{aligned}
$$

As $\varphi$ is an order preserving operator, by the theorem of Tarski [20], the set fix $(\varphi)$ is a complete lattice and then $(\mathcal{L}, \preceq)$ is also a complete lattice that is said to be $[6,7]$ the $L$-fuzzy concept lattice.

On the other hand, given $A \in L^{X}$, (or $B \in L^{Y}$ ) we can obtain the associated $L$-fuzzy concept applying twice the derivation operators. In the case of using a residuated implication, as we do in this work, the associated $L$-fuzzy concept is $\left(A_{12}, A_{1}\right)$ (or $\left.\left(B_{2}, B_{21}\right)\right)$.

Other important papers that generalize the Formal Concepts Analysis using residuated implication operators are due to R. Belohlavek $[4,5]$ and S. Pollandt [19]. Moreover, extensions of Formal Concept Analysis to the interval-valued case are in $[2,12,13]$ and to the fuzzy property-oriented and multi-adjoint concept lattices framework in $[15,16,17]$.

\section{Fuzzy context sequences}

In this section we are going to recover the fuzzy context sequences definition [1].

Definition 1 A fuzzy context sequence is a sequence of tuples $\left(L, X, Y, R_{i}\right), i=\{1, \ldots, n\}$, with $L=[0,1], X$ and $Y$ sets of objects and attributes respectively and $R_{i} \in L^{X \times Y}, \forall i=\{1, \ldots, n\}$, with $n \in \mathbb{N}$.

In the case of wanting to define a new fuzzy context that summarizes the information gathered in the sequence, we must aggregate the observations of the relations $R_{i}$. To this end, we could use the average of the values (with or without weight), obtain an interval-valued $L$-fuzzy context using the minimum and the maximum of the observations, or work with multivalued contexts. We have developed these ideas in previous works $[8,9]$.

The use of weighted averages $[10,11]$ to summarize the information stored in the different relations allows us to associate different weights with the $L$ fuzzy contexts highlighting some of them. Thus, the new relation $R$ is defined as:

$$
R(x, y)=\sum_{i=1}^{n} w_{i} \cdot R_{i}(x, y), \forall x \in X, y \in Y
$$

verifying, as is required by the definition, that $\sum_{i=1}^{n} w_{i}=1, i=\{1, \ldots, n\}$.

However, it is possible that some observations of a $L$-fuzzy context of the sequence be interesting whereas others not so much. For instance, as we studied in [3], the methods used for obtaining the fuzzy concepts do not give good results when we have very small values in some relations.

On the other hand, to study similar situations by means of multivalued contexts in [8] we used multisets and expertons. In that case, all the observations were analyzed globally without the study of temporality.

Let us see the following example.

Example 1 Let $\left(L, X, Y, R_{i}\right), i=\{1, \ldots, n\}$, be a fuzzy context sequence that represents the sales of sports articles $(X)$ in some establishments $(Y)$ throughout a period of time, and we want to study the places where the main sales hold taking into account that there are seasonal sporting goods (for instance skies, bathing suits) and of a certain zone (selling skies in Colorado is more possible than in Florida).

To analyze this situation, in [1] we use the OWA $[14,26]$ operators with the most of the weights near the largest values. In this way, we give more relevance to the largest observations, independently of the moment when they have taken place and, on the other hand, we would avoid some small values in the resulting relations (that can give problems 
in the calculation of the $L$-fuzzy concepts as it has already been studied in [3]).

These are the definitions of these operators given by Yager [26]:

Definition 2 A mapping $F_{w}$ from $L^{n} \longrightarrow L$, where $L=[0,1]$, is called an OWA operator of dimension $n$ if associated with $F_{w}$ is a weighting $n$-tuple $w=$ $\left(w_{1}, w_{2} \ldots w_{n}\right)$ such that $w_{i} \in[0,1]$ and $\sum_{1<i<n} w_{i}=1$, where $F_{w}\left(a_{1}, a_{2}, \ldots a_{n}\right)=w_{1} \cdot b_{1}+w_{2} . b_{2}+\cdots+w_{n} . b_{n}$, with $b_{i}$ the ith largest element in the collection $a_{1}, a_{2}, \ldots a_{n}$.

We applied these OWA operators to the fuzzy contexts to study the values that stand out in the fuzzy concepts and to analyze tendencies when the sequence represents the evolution in time.

Returning to the initial situation and using these OWA operators, in [1] we give the following definition that summarizes the information stored in the fuzzy context sequence:

Definition 3 Let $\left(L, X, Y, R_{i}\right), i=\{1, \ldots, n\}$, be the fuzzy context sequence and $F_{w}$ an $O W A$ aggregation operator. We can define a L-fuzzy relation $R_{F_{w}}$ that aggregates the information of the different $L$-fuzzy contexts, in the case that we want to study the largest values, by means of this expression:

$$
\begin{aligned}
R_{F_{w}}(x, y)= & F_{w}\left(R_{1}(x, y), R_{2}(x, y) \ldots R_{n}(x, y)\right)= \\
= & w_{1} \cdot b_{1}+w_{2} . b_{2}+\cdots+w_{n} . b_{n}, \\
& \forall x \in X, y \in Y
\end{aligned}
$$

where $w=\left(w_{1}, w_{2}, \ldots w_{n}\right)$ is the weighting tuple associated with $F_{w}$.

Fixed instant $h$ and using different weighting vectors, we have two relevant particular cases. In the first one we have the minimum of the relation values between each object and each attribute from instant $h$ and in the second one, the average of these observations:

Let $\left(L, X, Y, R_{i}\right), i=\{1, \ldots, n\}$, be a fuzzy context sequence with $L=[0,1], X$ and $Y$ sets of objects and attributes respectively and $R_{i} \in L^{X \times Y}$.

Relevant case 1 Fixed instant $h$, we define a $L$ fuzzy relation $R_{F_{w}}^{h}$ using an OWA operator $F_{w}$ with a weighting tuple $w$ of dimension $k=n-h+1$, with $w_{k}=1$ and $w_{i}=0, \forall i \neq k$ :

$$
\begin{aligned}
R_{F_{w}}^{h}(x, y)= & F_{w}\left(R_{h}(x, y), R_{h+1}(x, y) \ldots R_{n}(x, y)\right)= \\
& \min _{j \geq h}\left\{R_{j}(x, y)\right\}, \forall x \in X, y \in Y .
\end{aligned}
$$

Relevant case 2 Fixed instant $h$, we define a $L$ fuzzy relation $R_{F_{\hat{w}}}^{h}$ using an $O W A$ operator $F_{\hat{w}}$ with a weighting tuple $\hat{w}$ of dimension $k=n-h+1$ with $\hat{w}_{i}=1 / k, \forall i$ :

$$
\begin{aligned}
R_{F_{\hat{w}}}^{h}(x, y)= & F_{\hat{w}}\left(R_{h}(x, y), R_{h+1}(x, y) \ldots R_{n}(x, y)\right)= \\
& \sum_{j=h}^{n} \frac{R_{j}(x, y)}{k}, \forall x \in X, y \in Y .
\end{aligned}
$$

Example 2 The fuzzy context sequence $\left(L, X, Y, R_{i}\right), i=\{1, \ldots, 5\}$, represents the sports articles sales $X=\left\{x_{1}, x_{2}, x_{3}\right\}$ in some establishments $Y=\left\{y_{1}, y_{2}, y_{3}\right\}$ during a period of time. In the following relations $R_{i}$ that have values in $L=[0,1]$, the percentage of product sales in each establishment based on the stock during the last 5 months are gathered.

$$
\begin{gathered}
R_{1}=\left(\begin{array}{ccc}
0.7 & 1 & 0.8 \\
0 & 0.1 & 0.1 \\
0 & 0.1 & 0
\end{array}\right) R_{2}=\left(\begin{array}{ccc}
1 & 0.8 & 1 \\
0.2 & 0.4 & 0.1 \\
0 & 0 & 0.2
\end{array}\right) \\
R_{3}=\left(\begin{array}{ccc}
1 & 1 & 1 \\
0.6 & 0.5 & 0.7 \\
0 & 0.1 & 0.2
\end{array}\right) R_{4}=\left(\begin{array}{lll}
0.5 & 0.4 & 0.6 \\
0.1 & 0.5 & 0.3 \\
0.6 & 0.8 & 0.8
\end{array}\right) \\
R_{5}=\left(\begin{array}{ccc}
0.1 & 0 & 0 \\
0 & 0.1 & 0 \\
0.8 & 1 & 0.9
\end{array}\right)
\end{gathered}
$$

If we want to study tendencies of the sequence, we can consider a value $h$ and analyze the $L$-fuzzy concepts.

For instance, if $h=4$, using Relevant case 1 we have the $L$-fuzzy relation:

$$
R_{F_{w}}^{4}=\left(\begin{array}{ccc}
0.1 & 0 & 0 \\
0 & 0.1 & 0 \\
0.6 & 0.8 & 0.8
\end{array}\right)
$$

and, taking as $L$-fuzzy context $\left(L, X, Y, R_{F_{w}}^{4}\right)$ and using the Lukasiewicz implication to obtain the $L$ fuzzy concepts associated with the crisp singletons, we have the following results:

$$
\begin{aligned}
& \left\{x_{1}\right\} \longrightarrow\left(\left\{x_{1} / 1, x_{2} / 0.9, x_{3} / 1\right\},\left\{y_{1} / 0.1, y_{2} / 0, y_{3} / 0\right\}\right) \\
& \left\{x_{2}\right\} \longrightarrow\left(\left\{x_{1} / 0.9, x_{2} / 1, x_{3} / 1\right\},\left\{y_{1} / 0, y_{2} / 0.1, y_{3} / 0\right\}\right) \\
& \left\{x_{3}\right\} \longrightarrow\left(\left\{x_{1} / 0.2, x_{2} / 0.2, x_{3} / 1\right\},\left\{y_{1} / 0.6, y_{2} / 0.8, y_{3} / 0.8\right\}\right)
\end{aligned}
$$

However, if we use Relevant case 2, we obtain:

$$
R_{F_{\hat{w}}}^{4}=\left(\begin{array}{ccc}
0.3 & 0.2 & 0.3 \\
0.05 & 0.3 & 0.15 \\
0.7 & 0.9 & 0.85
\end{array}\right)
$$

and, taking as $L$-fuzzy context $\left(L, X, Y, R_{F_{\hat{w}}}^{4}\right)$ :

$$
\begin{aligned}
& \left\{x_{1}\right\} \longrightarrow\left(\left\{x_{1} / 1, x_{2} / 0.75, x_{3} / 1\right\},\left\{y_{1} / 0.3, y_{2} / 0.2, y_{3} / 0.2\right\}\right) \\
& \left\{x_{2}\right\} \longrightarrow\left(\left\{x_{1} / 0.9, x_{2} / 1, x_{3} / 1\right\},\left\{y_{1} / 0.05, y_{2} / 0.3, y_{3} / 0.15\right\}\right) \\
& \left\{x_{3}\right\} \longrightarrow\left(\left\{x_{1} / 0.3, x_{2} / 0.3, x_{3} / 1\right\},\left\{y_{1} / 0.7, y_{2} / 0.9, y_{3} / 0.85\right\}\right)
\end{aligned}
$$

In both cases, we can say that the future tendency is that only article $x_{3}$ will have good sales in all the establishments whereas $x_{1}$ and $x_{2}$ will not be sold much and always associated with $x_{3}$, the first one in the establishment $y_{1}$ essentially, and the second one in $y_{2}$.

We can observe that in all the cases the membership degrees of the attributes using the average are greater than using the minimum. 
Obviously, the smaller is the value of $h$, the safer will be the prediction that we do.

However, there is a problem in the definition of $R_{F_{w}}^{h}$ and $R_{F_{\hat{w}}}^{h}$ : all the values from instant $h$ are considered equally valid. But, if we are analyzing the evolution in time, the closest to the current instant values should be the most important ones.

On the other hand, the values previous to instant $h$ are not taken into account. For instance, in Example 2 can be seen that $x_{1}$ has not high sales in any establishment, however, if we look at the evolution in time of $x_{1}$ then we can see that sales in all the establishments have been good except in the two last months. The WOWA operators allow us to perform this assessment.

\section{Evolution in time of the fuzzy context sequence}

In order to study the evolution in time of these sequences, we are going to use WOWA operators [22].

The Weighted OWA operators (WOWA) were defined by Torra [22] and combine the advantages of the OWA operators and the ones of the weighted mean. These operators consider two weighting vectors: $w=\left(w_{1}, w_{2}, \ldots w_{n}\right)$ corresponding to the relevance of the values (OWA operator) and $p=$ $\left(p_{1}, p_{2} \ldots p_{n}\right)$ corresponding to the relevance of the sources or experts. In the case of fuzzy context sequences that evolve in time, we will use $p$ to assign a weight for each $L$-fuzzy context depending on the distance to the present time.

As particular cases, if $w_{i}=1 / n, \forall i$ then we have the weighted mean with $p$ and if $p_{i}=1 / n, \forall i$, an OWA operator with $w$.

This is the definition [22]:

Definition 4 Let $p, w$ be weighting vectors of dimension $n, p=\left(p_{1}, p_{2}, \ldots p_{n}\right)$ and $w=$ $\left(w_{1}, w_{2}, \ldots w_{n}\right)$ such that $p_{i}, w_{i} \in[0,1]$ and $\sum_{i} p_{i}=$ $\sum_{i} w_{i}=1$.

In this case, a mapping $F_{p w}: \mathbb{R}^{n} \longrightarrow \mathbb{R}$ is a Weighted Ordered Weighted Averaging (WOWA) operator of dimension $n$ if

$$
F_{p w}\left(a_{1}, \ldots, a_{n}\right)=\sum_{i} \omega_{i} a_{\sigma(i)}
$$

where $\{\sigma(1), \ldots, \sigma(n)\} \quad$ is a permutation of $\{1, \ldots, n\}$ such that $a_{\sigma(i-1)} \geq a_{\sigma(i)}$ for all $i=\{2, \ldots, n\}$ (i.e., $a_{\sigma(i)}$ is the ith largest element in the collection $\left.a_{1}, \ldots, a_{n}\right)$, and the weight $\omega_{i}$ is defined as

$$
\omega_{i}=w^{*}\left(\sum_{j \leq i} p_{\sigma(j)}\right)-w^{*}\left(\sum_{j<i} p_{\sigma(j)}\right)
$$

with $w^{*}$ a monotone increasing function that interpolates the points $\left(i / n, \sum_{j \leq i} w_{j}\right)$ together with the point (0,0). $w^{*}$ is required to be a straight line when the points can be interpolated in this way.

\subsection{Relations obtained setting an instant $h$ and using WOWA operators}

We can perform a comparative study using different instants $h$ as the one done in the previous section. The following results show that relations defined in Section 3 can be obtained as a particular case when we use WOWA operators:

Proposition 1 Fixed instant $h$, consider $k=n-$ $h+1$ and any weighting vector $p$,

a) Consider $w=\left(w_{1}, w_{2}, \ldots w_{n}\right)$ such that:

$$
w_{i}= \begin{cases}1 & \text { if } i=k \\ 0 & \text { in other case }\end{cases}
$$

If we take as interpolation function $w^{*}$ such that

$$
w^{*}(z)= \begin{cases}0 & \text { if } z<1 \\ 1 & \text { in other case }\end{cases}
$$

Then, $R_{F_{p w}}(x, y)=R_{F_{w}}^{h}(x, y), \forall x \in X, \forall y \in Y$, is the minimum of the $k$ considered observations.

b) If $\hat{w}$ such that $w_{i}=1 / k, \forall i$, we can take as interpolation function $\hat{w}^{*}$ such that $\hat{w}^{*}(z)=$ $z, \forall z \in[0,1]$.

Then, $R_{F_{p \hat{w}}}(x, y)=R_{F_{p}}^{h}(x, y), \forall x \in X, \forall y \in Y$, is the weighting mean with a weighting vector p.

Proof 1 a) If we take as interpolation function $w^{*}$ then, we obtain $\omega_{i}=0, \forall i<k$ and $\omega_{k}=1$. Then, $R_{F_{p w}}(x, y)=\sum_{i} \omega_{i} R_{\sigma_{x y}(i)}(x, y)=$ $R_{F_{w}}^{h}(x, y), \forall x \in X, \forall y \in Y$, is the minimum of the $k$ considered observations.

b) If we take as interpolation function $\hat{w}^{*}$ such that $\hat{w}^{*}(z)=z, \forall z \in[0,1]$, and $\sigma_{x y}$ is the permutation that orders $\left(R_{h}(x, y), R_{h+1}(x, y), \ldots R_{n}(x, y)\right)$, $\forall x \in X, \forall y \in Y$. Hence, $\omega_{i}=p_{\sigma_{x y}(i)}, \forall i \leq$ $k, \forall x \in X, \forall y \in Y$.

Then, $\quad R_{F_{p \hat{w}}}(x, y)=\sum_{i} \omega_{i} R_{\sigma_{x y}(i)}(x, y)=$ $R_{F_{p_{\sigma_{x y}(i)}}}^{h}(x, y), \forall x \in X, \forall y \in Y$, is the weighting mean with a weighting vector $p$.

We have to take into account that for any $w$, the use of weighting vectors $p$ of dimension $n$ with null values up to the instant $h$ does not go well since the values of $p$ are reordered with permutation $\sigma$.

On the other hand, considering the fuzzy context sequence $\left(L, X, Y, R_{i}\right), i=\{1, \ldots, n\}$, an interesting result is obtained fixing different instants: 
Proposition 2 Consider $k<l$. We can take the weighting vectors $w^{k}$ and $w^{l}$ such that:

$$
w_{i}^{\alpha}= \begin{cases}1 & \text { if } i=\alpha \\ 0 & \text { in other case }\end{cases}
$$

If we take as interpolation functions respectively

$$
w^{\alpha *}(z)= \begin{cases}0 & \text { if } z<\alpha / n \\ 1 & \text { in other case }\end{cases}
$$

the WOWA operators $F_{w^{k}}$ and $F_{w^{l}}$ can be taken and we can prove that $R_{F_{w^{k}}} \leq R_{F_{w^{l}}}$.

Proof 2 In the conditions of the theorem $\omega_{i}^{k} \leq$ $\omega_{i}^{l}, \forall i=\{1, \ldots, n\}$. So, $R_{F_{w^{k}}} \leq R_{F_{w l} l}$.

\subsection{Aggregating the fuzzy context sequence using WOWA operators}

As we have said in the study of previous section, we establish an instant $h$ from which we look at the observations and the values of previous contexts are not taken into account. By this reason, in some situations the obtained results can be improved.

If we take $h$ close to $n$ and come back to the example, it seems that there is no $x_{1}$ sales in any establishment. However, if we look at the context sequence, we see that it has been. On the other hand, if $h$ is close to 1 , we are considering values which happened a long time ago and may not have as much relevance as the recent ones for the study of trends.

The use of WOWA operators will allow to perform a proper treatment of the sequence better than the proposed in the previous section. We can define a $L$-fuzzy relation $R_{F_{p w}}$ that aggregates the information of the different fuzzy contexts, by means of this expression:

Definition 5 Let $\left(L, X, Y, R_{i}\right), i=\{1, \ldots, n$,$\} be$ the fuzzy context sequence and $F_{p w}$ an WOWA aggregation operator with $p=\left(p_{1}, p_{2}, \ldots p_{n}\right)$ and $w=\left(w_{1}, w_{2}, \ldots w_{n}\right)$ such that $p_{i}, w_{i} \in[0,1]$ and $\sum_{i} p_{i}=\sum_{i} w_{i}=1$. Then,

$$
\begin{aligned}
R_{F_{p w}}(x, y)= & F_{p w}\left(R_{1}(x, y), \ldots R_{n}(x, y)\right)= \\
& \sum_{i} \omega_{x y_{i}} R_{\sigma_{x y}(i)}(x, y)
\end{aligned}
$$

where for every $(x, y)$ we have $\sigma_{x y}=$ $\left\{\sigma_{x y}(1), \ldots, \sigma_{x y}(n)\right\}$ a permutation of $\{1, \ldots, n\}$ such that $R_{\sigma_{x y}(i-1)}(x, y) \geq R_{\sigma_{x y}(i)}(x, y)$ for all $i=\{2, \ldots, n\}$, and the weighting vector $\omega_{x y}$ defined in Definition 4.

We are defining a single $R_{F_{p w}}$ combining all the contexts of the sequence with two tables of weights. We consider interesting to use the weighting vector $p$ to give more relevance to the most recently contexts. On the other hand, we will take as a vector of weights $w$ one that will allow to highlight membership degrees close to 1 since we want to study the largest values.

Relevant case 3 We define $R_{F_{p w}}$ using $p$ and $w$ such that

$$
p_{i}=\frac{2 i}{n(1+n)}, w_{i}=\frac{2(n-i+1)}{n(1+n)}, \forall i=\{1, \ldots, n\}
$$

Example 3 In our example $n=5$ and we have $p=(1 / 15,2 / 15,3 / 15,4 / 15,5 / 15)$ and $w=$ $(5 / 15,4 / 15,3 / 15,2 / 15,1 / 15)$.

We will use the following function to interpolate $(0,0),\left(\frac{1}{5}, \frac{5}{15}\right),\left(\frac{2}{5}, \frac{9}{15}\right),\left(\frac{3}{5}, \frac{12}{15}\right),\left(\frac{4}{5}, \frac{14}{15}\right),(1,1)$ :

$$
w^{*}(x)= \begin{cases}\frac{5}{3} x & \text { if } 0 \leq x<\frac{1}{5} \\ \frac{4}{3} x+\frac{1}{15} & \text { if } \frac{1}{5} \leq x<\frac{2}{5} \\ x+\frac{1}{5} & \text { if } \frac{2}{5} \leq x<\frac{3}{5} \\ \frac{2}{3} x+\frac{2}{5} & \text { if } \frac{3}{5} \leq x<\frac{4}{5} \\ \frac{1}{3} x+\frac{2}{3} & \text { if } \frac{4}{5} \leq x \leq 1\end{cases}
$$

Using this function, we obtain a vector $\omega_{x y}$ for each pair object-attribute $(x, y)$ such that following definition given in the last relevant case, we obtain:

$$
R_{F_{p w}}=\left(\begin{array}{ccc}
0.71 & 0.67 & 0.62 \\
0.26 & 0.41 & 0.35 \\
0.58 & 0.76 & 0.73
\end{array}\right)
$$

In this case, the $L$-fuzzy concepts obtained from the basic points are:

$$
\begin{aligned}
& \left\{x_{1}\right\} \longrightarrow\left(\left\{x_{1} / 1, x_{2} / 0.55, x_{3} / 0.87\right\},\left\{y_{1} / 0.71, y_{2} / 0.67, y_{3} / 0.62\right\}\right) \\
& \left\{x_{2}\right\} \longrightarrow\left(\left\{x_{1} / 1, x_{2} / 1, x_{3} / 1\right\},\left\{y_{1} / 0.26, y_{2} / 0.41, y_{3} / 0.35\right\}\right) \\
& \left\{x_{3}\right\} \longrightarrow\left(\left\{x_{1} / 0.89, x_{2} / 0.62, x_{3} / 1\right\},\left\{y_{1} / 0.58, y_{2} / 0.75, y_{3} / 0.73\right\}\right)
\end{aligned}
$$

We can say that the membership degrees of the attributes in the $L$-fuzzy concepts associated with $x_{1}$ are greater than the ones obtained using $R_{F_{w}}^{4}$ and $R_{F_{\hat{w}}}^{4}$ since the observations of the first contexts (high values) are taken into account in this case. However, the membership degrees of $x_{3}$ have decreased slightly (also by the influence of the first contexts).

The obtained results using WOWA operators can be better than the previous ones because take into account all the contexts of the sequence.

\section{Conclusions and future work}

In this work, we have used WOWA operators to study fuzzy context sequences. When these sequences represent the evolution in time of a $L$-fuzzy context, this study can be interpreted as a tendencies estimation.

To do this, we have studied the derived information by means of the $L$-fuzzy concepts. Although WOWA operators have a bad behavior in some concrete cases, in the fuzzy context sequences can improve the previous results obtained. 
These fuzzy contexts that evolve with time can be generalize when $L$ is a complete lattice. Our aim is the study of this situation with appropriate WOWA operators.

\section{Acknowledgments}

This paper is partially supported by the Research Group "Intelligent Systems and Energy (SI+E)" of the Basque Government, under Grant IT677-13, and by the Research Group "Artificial Intelligence and Approximate Reasoning" of the Public University of Navarra, under TIN2013-40765-P.

\section{References}

[1] C. Alcalde, A. Burusco, R. Fuentes-González, The study of fuzzy context sequences, International Journal of Computational Intelligence Systems, 6 (3):518-529, 2013.

[2] C. Alcalde, A. Burusco, R. Fuentes-González, I. Zubia, Treatment of $L$-fuzzy contexts with absent values, Information Sciences, 179 (1-2):115, 2009.

[3] C. Alcalde, A. Burusco, R. Fuentes-González, I. Zubia, The use of linguistic variables and fuzzy propositions in the L-Fuzzy Concept Theory, Computers and Mathematics with Applications, 62:3111-3122, 2011.

[4] R. Bělohlávek, Fuzzy Galois Connections, Math. Logic Quarterly, 45 (4):497-504, 1999.

[5] R. Bělohlávek, V. Vychodil, What is a fuzzy concept lattice. In Proceedings of the Third International Conference on Concept Lattice and their Applications, CLA 2005, Olomouc, Czech Republic, pages 34-45, 2005.

[6] A. Burusco, R. Fuentes-González, The Study of the L-Fuzzy Concept Lattice, Mathware and Soft Computing, 1 (3):209-218, 1994.

[7] A. Burusco, R. Fuentes-González, Construction of the L-Fuzzy Concept Lattice, Fuzzy Sets and Systems, 97 (1):109-114, 1998.

[8] A. Burusco, R. Fuentes-González, Contexts with multiple weighted values, The International Journal of Uncertainty, Fuzziness and Knowledge-based Systems, 9 (3):355-368, 2001.

[9] A. Burusco, R. Fuentes-González, The study of the interval-valued contexts, Fuzzy Sets and Systems, 121:69-82, 2001.

[10] T. Calvo, R. Mesiar, Weighted triangular norms-based aggregation operators, Fuzzy Sets and Systems, 137:3-10, 2003.

[11] T. Calvo, R. Mesiar, Aggregation operators: ordering and bounds, Fuzzy Sets and Systems, 139:685-697, 2003.

[12] Y. Djouadi, H. Prade, Interval-Valued Fuzzy Galois Connections: Algebraic Requirements and Concept Lattice Construction, Fundamenta Informaticae, 99 (2):169-186, 2010.
[13] Y. Djouadi, H. Prade, Possibility- theoretic extension of derivation operators in formal concept analysis over fuzzy lattices, FODM 10 (4):287309, 2011.

[14] J. Fodor, J. L. Marichal, M. Roubens, Characterization of the Ordered Weighted Averaging Operators, IEEE Transactions on Fuzzy Systems, 3 (2):236-240, 1995.

[15] J. Medina, Multi-adjoint property-oriented concept lattices, Information Sciences, 190:95106, 2012.

[16] J. Medina, M. Ojeda-Aciego, Multi-adjoint t-concept lattices, Information Sciences, 180 (5):712-725, 2010.

[17] J. Medina, M. Ojeda-Aciego, Dual multiadjoint concept lattices, Information Sciences, 225:47-54, 2013.

[18] R. Neouchi, A. Y. Tawfik, R. A. Frost, Towards a Temporal Extension of Formal Concept Analysis. In Proceedings of Canadian Conference on Artificial Intelligence, pages 335-344, 2001.

[19] S. Pollandt, Fuzzy Begriffe, Springer, 1997.

[20] A. Tarski, A lattice theoretical fixpoint theorem and its applications. Pacific Journal of Mathematics. 5, pp.285-310, 1955.

[21] A. Y. Tawfik, G. Scott, Temporal Matching under Uncertainty. In Proceedings of the Eighth International Workshop on Artificial Intelligence and Statistics, 2001.

[22] V. Torra, The weighted OWA Operator, International Journal of Intelligennt Systems, 12:53166, 1997.

[23] R. Wille, Restructuring lattice theory: an approach based on hierarchies of concepts. In $R i$ val I. (Ed.), Ordered Sets, Reidel, DordrechtBoston, pages 445-470, 1982.

[24] K. E. Wolff, States, Transitions, and Life Tracks in Temporal Concept Analysis, Formal Concept Analysis, pages 127-148, 2005.

[25] K. E. Wolff, Temporal Relational Semantic Systems, ICCS, pages 165-180, 2010.

[26] R. R. Yager, On ordered weighted averaging aggregation operators in multi-criteria decision making, IEEE Transactions on Systems, Man and Cibernetics, 18:183-190, 1988. 